\title{
The role of the medial prefrontal cortex in attention: Altering predictability of task difficulty
}

\author{
J. M. WILLIAMS, E. G. MOHLER, and B. GIVENS \\ Ohio State University, Columbus, Ohio
}

\begin{abstract}
Rats were trained on a signal detection task in which a light ( 200 or $500 \mathrm{msec})$ appeared randomly over one of three levers. The rat was required to press the illuminated lever. There were two versions of the task: a light detection-random (LDR) and a light detection-block (LDB) task that differed only in the predictability of the difficulty level of the upcoming stimuli (LDR = unpredictable; LDB = predictable). Scopolamine infusions into the anterior cingulate and the prelimbic cortex significantly impaired choice accuracy in the LDR, but not in the LDB task. However, saline infusions into the prelimbic cortex also decreased choice accuracy, suggesting that the prelimbic cortex is particularly sensitive to disruption under conditions of high attentional demand. Overall, the results indicate that the prefrontal cortex is involved in mediating performance on tasks in which the difficulty level is unpredictable, thus requiring more focused attention throughout the task.
\end{abstract}

Numerous studies support the hypothesis that the medial prefrontal cortex (mPFC) is actively involved in attentional processing (Herremans, Hijzen, Welborn, Olivier, \& Slangen, 1996; Muir, Everitt, \& Robbins, 1996), especially in maintaining attentional focus when task difficulty is increased by experimental manipulations, such as the addition of distracting stimuli (Chao \& Knight, 1995). More specifically, it has been hypothesized that the mPFC can modulate its activity up or down to correspond to the level of task difficulty. On a task requiring subjects to match a sample stimulus with previously occurring stimuli, EEG/MRI analyses revealed that a midline frontal theta rhythm was responsive to changes in both task difficulty (as assessed by increased memory load) and the amount of practice on a task. This change in midline frontal theta rhythm was localized to the anterior cingulate (AC) region (Gevins, Smith, McEvoy, \& Yu, 1997). Medial prefrontal neuronal activity has also been correlated with the difficulty level of cognitive tasks in nonhuman primates. A significant percentage ( $15 \%$ ) of $\mathrm{mPFC}$ neurons were found to modulate their activity across testing sessions as the level of task difficulty was either increased or decreased (Lecas, 1995).

The mPFC is involved in tasks that require controlled attentional processes - that is, tasks that are difficult or require the subject to actively focus on a stimulus. In contrast, tasks that are overlearned and involve attentional performance that has become automatic do not appear to engage the mPFC (for a review, see Passingham, 1996). Positron emission tomography (PET) studies have shown

Correspondence concerning this article should be addressed to B. Givens, Department of Psychology, Ohio State University, Room 33, Townshend Hall, Columbus, OH 43210 (e-mail: givens+@osu.edu). that the mPFC is activated when a subject first learns a motor task consisting of eight sequential finger movements. However, once the task becomes overlearned and the attentional demands shift from controlled to more automatic levels of processing, the mPFC becomes disengaged (Jenkins, Brooks, Nixon, Frackowiak, \& Passingham, 1994; Jueptner et al., 1997). If the experimenter instructs subjects to use more controlled processes by actively focusing their attention on performing an overlearned task, the mPFC again becomes active (Jueptner et al., 1997). This pattern of prefrontal PET activity has also been found in verb generation tasks (Petersen, Van Mier, Fiez, \& Raichle, 1998), Stroop test paradigms (Pardo, Pardo, Janer, \& Raichle, 1990), and divided attention tasks requiring attention to multiple stimulus attributes (Corbetta, Miezin, Dobmeyer, Shulman, \& Petersen, 1991).

Although the research in humans has largely focused on the role of the $\mathrm{AC}$ region, animal studies have implicated other areas of the $\mathrm{MPFC}$, such as the prelimbic (PL) region, in cognitive processes such as spatial working memory (Delatour \& Gisquet-Verrier, 1996; Ragozzino, Adams, \& Kesner, 1998) and executive decisional processing (Miner, Ostrander, \& Sarter, 1997). In studies that have directly assessed the involvement of both the $\mathrm{AC}$ and the $\mathrm{PL}$ in cognitive processing, dissociations in function between these two areas of the MPFC have been found (Ragozzino et al., 1998), with the PL being more involved in spatial working memory than the pre-genu AC. In addition, prefrontal dissociations have also been found following lesions to the medial prefrontal cortex (which includes the PL cortex, as well as the pre-genu AC and Fr2) and the post-genu AC (Bussey, Muir, Everitt, \& Robbins, 1997; Muir et al., 1996). For instance, Muir et al. (1996) found that, on a five-choice serial reaction time task, post-genu 
$\mathrm{AC}$ lesions increased premature responding, whereas medial prefrontal lesions decreased choice accuracy and increased perseverative responding and latency to respond. These studies suggest that the prefrontal cortex is involved in cognitive processing and that distinct subregions of the prefrontal cortex may underlie different aspects of cognitive processing.

One possible mechanism underlying the $\mathrm{mPFC}$ contributions to cognitive processing is the activation of the cholinergic system that ascends from the basal forebrain. Infusions of the cholinergic antagonist scopolamine into the mPFC impaired performance on a delayed conditional discrimination task (Herremans, Hijzen, \& Olivier, 1997). These scopolamine-induced impairments were reversed by local coadministration of the acetylcholinesterase inhibitor physostigmine. Scopolamine infusions into the prelimbic area also impaired working memory on delayed match-to-sample and delayed nonmatch-to sample tasks (Granon, Poucet, Thinus-Blanc, Changeux, \& Vidal, 1995). In addition, increases in acetylcholine efflux were seen in a signal detection task when the attentional demands were increased by introducing a flashing houselight distractor (Sarter et al., 1996). In this same study, a population of MPFC neurons whose activity increased during the presence of the distractor was identified. Lesions to the nucleus basalis of Meynert, which provides the major source of cholinergic innervation of the cerebral cortex (Mesulam, Mufson, Levey, \& Wainer, 1983), disrupts attentional performance (McGaughy, Kaiser, \& Sarter, 1996; Muir, Everitt, \& Robbins, 1994). Overall, these studies support the hypothesis that the mPFC cholinergic system plays a significant role in cognitive processing.

The studies above all stress a role for the mPFC in tasks requiring a subject to actively attend to incoming sensory information. Accordingly, the present study was designed to examine (1) the role of the MPFC in controlled versus automatic processing by assessing whether this dissociation applies to two tasks that differ only in the subjects' ability to predict the difficulty level of an upcoming stimulus, (2) possible dissociations between the post-genu $\mathrm{AC}$ and PL subregions of the $\mathrm{MPFC}$ in automatic versus controlled types of processing, and (3) possible cholinergic involvement in mediating $\mathrm{mPFC}$ contributions to controlled versus automatic processing by utilizing intracranial infusions of the muscarinic antagonist scopolamine.

\section{METHOD}

\section{Subjects}

Fourteen male Long-Evans rats were used in this experiment. The animals were housed in a colony room maintained at a constant temperature on a 12:12-h light:dark cycle, with the light period beginning at 7:00 a.m. All the experiments were conducted from 8:00 to 11:00 a.m. during the light cycle. At the start of training, the body weight of each rat was decreased to approximately $85 \%$ of the rat's ad-lib weight by restricting the rat's water intake. During training, the rats were given an unlimited amount of food and a sufficient amount of water to maintain the restricted body weight. The rats were assigned to a light detection-block (LDB) group $(n=6)$ or a light detection-random (LDR) group $(n=8)$.

\section{Apparatus}

Each rat was trained in one of eight operant chambers. Each operant chamber was placed in a sound-attenuating shell $(64 \times 41 \times$ $41 \mathrm{~cm}$ ) and consisted of two side walls of $0.5-\mathrm{cm}$ clear Plexiglas, front and back panels of stainless steel, and a floor consisting of parallel stainless steel rods, $1 \mathrm{~cm}$ apart. The front panel of the apparatus consisted of three levers, $7.5 \mathrm{~cm}$ above the floor. A circular light, $2.5 \mathrm{~cm}$ in diameter and $12 \mathrm{~cm}$ above the floor, was located above each lever. A central houselight, $18 \mathrm{~cm}$ above the floor, was located centrally on the front panel, and a tone generator, $12 \mathrm{~cm}$ above the floor, was on the back panel. A water dispenser, $2 \mathrm{~cm}$ above the floor in the center of the back panel, delivered a $0.08-\mathrm{ml}$ drop of water following rewarded leverpresses. The operant chambers were interfaced to a personal computer that controlled all behavioral acquisition and analysis with software developed by Med Associates, Inc. (East Fairfield, VT).

\section{Design and Procedure}

Shaping Step 1. Initially, the rats were trained to press levers to receive a water reward. Left, right, or center leverpresses were rewarded with a drop of water. To prevent the development of a bias toward one lever, the total number of presses on one lever could not exceed those to any of the remaining levers by more than five. If this occurred, subsequent responding to the more frequently pressed lever was no longer rewarded, until the imbalance was corrected. All the rats, regardless of group assignment, received the same shaping procedures in Shaping Step 1. The rats were trained in this step daily for $1-h$ sessions until they reached a criterion of 50 bar presses in $1 \mathrm{~h}$, after which they were moved to Shaping Step 2.

Shaping Step 2. The rats were trained on a three-choice signal detection task. At the beginning of each trial, the left, right, or center stimulus light was randomly illuminated. The rat was required to press the lever beneath the illuminated light in order to obtain a water reward. Incorrect responses were not rewarded. The stimulus remained lit until the rat made a lever response or $3 \mathrm{sec}$ had elapsed, whichever came first. After a randomly determined $12 \pm 3 \mathrm{sec}$ intertrial interval, the next trial was initiated. All the rats, regardless of group assignment, received the same shaping procedures in Shaping Step 2. The rats were trained in this step 6 days per week ( 120 trials per day) until they reached criteria of $80 \%$ choice accuracy and less than $30 \%$ omissions over 3 consecutive days, after which they were moved to Shaping Step 3. This criteria remained the same for Shaping Step 3.

Shaping Step 3. At the start of each trial, the left, right, or center stimulus was randomly illuminated for either 500 or $1,000 \mathrm{msec}$. The rat was required to press the lever beneath the illuminated stimulus in order to obtain a water reward. Incorrect responses were not rewarded. If the rat did not respond within $3 \mathrm{sec}$ of stimulus onset, the trial was terminated, and a no response was recorded. A variable $12 \pm 3 \mathrm{sec}$ interval separated each trial.

LDB rats were presented with 120 trials broken into four discrete blocks of 30 trials. Each block consisted of only one stimulus length (i.e., all 500-msec trials or all 1,000-msec trials). The rats received two blocks of trials at each stimulus length in one of two sequences (500-1,000-500-1,000 or 1,000-500-1,000-500). LDR rats were tested on a signal detection task similar to the LDB task, with one major exception. Unlike the LDB task, the 120 daily trials were not divided into four discrete blocks, and stimulus lengths (also 500 and $1,000 \mathrm{msec}$ ) were randomly determined for each individual trial. After reaching criteria, the rats were moved to the final training program.

Final training program. For the final training step, the stimulus lengths were lowered from 500 and $1,000 \mathrm{msec}$ to 200 and $500 \mathrm{msec}$. 
The rats were trained on this final program until they reached criteria of $75 \%$ choice accuracy and less than $30 \%$ omissions over 3 consecutive days, at which point they were surgically implanted with guide cannulae into the prefrontal cortex.

\section{Surgery}

Surgeries were performed in aseptic conditions under sodium pentobarbital anesthesia $(45 \mathrm{mg} / \mathrm{kg})$. The body temperature of each rat was monitored during surgery and maintained at approximately $36^{\circ} \mathrm{C}$ with a thermal blanket (Harvard Apparatus, South Natick, MA). Supplemental injections of sodium pentobarbital $(15 \mathrm{mg} / \mathrm{kg}$ ) were administered if corneal, hindlimb, or tail reflexes or rapid respiratory rates were present.

Guide cannulae made of 26-gauge stainless steel tubing (13 mm in length) were implanted bilaterally into the post-genu AC region of the prefrontal cortex $(0.7 \mathrm{~mm}$ anterior to bregma, $1.0 \mathrm{~mm}$ lateral to midline, and $1.3 \mathrm{~mm}$ ventral to the dural surface; $15^{\circ}$ angle medially) and bilaterally into the PL region of the prefrontal cortex (2.7 $\mathrm{mm}$ anterior to bregma, $1.5 \mathrm{~mm}$ lateral to midline, and $2.8 \mathrm{~mm}$ ventral to the dural surface; $15^{\circ}$ angle medially). The cannulae were secured with dental acrylic to four small screws that were placed in the skull (three screws were placed posterior to bregma, and one screw was placed anterior to bregma). A stylet made of 32-gauge stainless steel tubing ( $14 \mathrm{~mm}$ in length with a $1-\mathrm{mm}$ bent tip) was placed into each guide cannulae to prevent clogging. Mycitracin Plus (an antibiotic/local anesthetic) was placed around the dental acrylic to prevent infections and to reduce discomfort.

Following surgery, the rats were given food and water ad lib for 3 days, after which they were gradually returned to their initial state of water restriction while their weight, posture, and locomotor activity were monitored to ensure full recovery. Testing resumed 1 week following surgery.

\section{Postsurgery Testing}

After reestablishing baseline performance, the rats received drug infusions into the post-genu AC and the PL to determine the involvement of these structures in attentional processing. Each rat was brought to the testing room in its home cage. The stylets were removed, and a sterile injector was placed into each guide cannula. The injector was connected to the microinfusion syringe pump with polythene tubing. The syringe pump was programmed to deliver fluid at $0.2 \mu \mathrm{l} / \mathrm{min}$ over a $2.5-\mathrm{min}$ time period. The rats were infused with either $0.5 \mu \mathrm{l}$ of saline or $0.5 \mu \mathrm{l}$ of scopolamine (either $5-\mu \mathrm{g}$ or 10 $\mu \mathrm{g}$ doses). Each LDR rat received all three infusion conditions into both the AC and the PL, for a total of three infusions into each brain area. Each LDB rat received all three infusion conditions in both brain locations under two different block conditions (200-500 200-500 and 500-200-500-200 stimulus length orders), for a total of six infusions into each brain area. The scopolamine solutions were prepared in saline. Infusions were made every 3 rd day, alternating between $\mathrm{AC}$ and PL sites. The rats continued to receive training on noninfusion days to maintain stable task performance. The order of drug presentation, site of injection, and stimulus order (for LDB rats ) was counterbalanced throughout the study.

\section{Histology}

To verify the accuracy of the cannulae placements and to ascertain the extent of the drug infusions, the rats were deeply anesthetized with sodium pentobarbital and infused with Chicago sky blue dye $(2 \% ; 0.5 \mu \mathrm{l})$ to mark the location of the placements. Following the dye infusion, each rat was perfused transcardially first with saline $(200 \mathrm{ml})$ and then with a $10 \%$ formalin solution $(200 \mathrm{ml})$. The brains were removed, sectioned $(40 \mu \mathrm{m})$, and mounted on gelatin-coated slides. Cannula placement was determined by examining dye diffusion and observable cannula tracks.

\section{Analysis}

Three rats were unable to complete testing because of illness and were removed from the study. All the subsequent analyses pertain to the remaining 11 rats (LDB: $n=6$; LDR: $n=5$ ). A mixed design analysis of variance (ANOVA) was used to analyze postsurgery data, using drug, infusion area, and stimulus length as repeated factors and group designation (LDB or LDR) as a between-subjects factor. The dependent variables were overall percent accuracy, omission rate, and latency to respond.

\section{RESULTS}

\section{Histology}

All the rats included in the subsequent analyses had bilateral implants into the PL and AC cortices, as determined by location of dye infusion and cannula tracks. All the rats had evidence of gliosis surrounding the infusion site. The top panel of Figure 1 represents the projected injector tip placements in the PL. Although the initial coordinate for the PL cannulae placements was $2.70 \mathrm{~mm}$ anterior to bregma, the heaviest concentration of injector tip placements/cannulae tracks was at $3.20 \mathrm{~mm}$ anterior to bregma. Accordingly, dye for the PL injector tip placements was also centered approximately $3.20 \mathrm{~mm}$ anterior to bregma ( $n=9$; Figure 1$)$. The remaining 2 rats had cannula placements centered at 3.70 and $2.20 \mathrm{~mm}$ anterior to bregma, also falling within the PL. Seven rats had bilateral injector tip placements in the PL area of the mPFC. All cannulae placements were rostral to the genu of the corpus callosum. One rat had bilateral injector tip placements in the infralimbic cortex (IL), 2 rats had one tip in the PL and one in the IL, and 1 rat had one tip in the PL and one in the AC. All cases, regardless of tip placement, involved the spread of the dye into the PL.

The bottom panel of Figure 1 represents injector tip placement into the AC. The heaviest concentration of dye for the AC cannula placements was centered approximately $1.00 \mathrm{~mm}$ anterior to bregma $(n=9$; Figure 1$)$. The remaining 2 rats had cannula placements at 1.60 and $1.70 \mathrm{~mm}$ anterior to bregma. With the exception of the latter cannulae placement, all the rats had bilateral injector tip placements in the post-genu AC. The placement $1.70 \mathrm{~mm}$ anterior to bregma was situated slightly anterior to the genu of the corpus callosum, but an analysis of the spread of the dye infusion revealed spread of the dye to the post-genu areas of the $\mathrm{AC}$, but no significant encroachment upon the PL.

\section{Overall Choice Accuracy Analysis}

Overall, choice accuracy at the $500-\mathrm{msec}$ stimulus length was significantly better than choice accuracy at the 200 -msec stimulus length [main effect of stimulus length: $F(1,9)=109.458, p<.05]$, suggesting that the LDB and LDR tasks tapped into the ability of the rats to detect, attend to, and respond to visually presented stimuli. An analysis of the effects of infusions on choice accuracy also revealed a significant infusion effect $[F(3,27)=13.039$, 

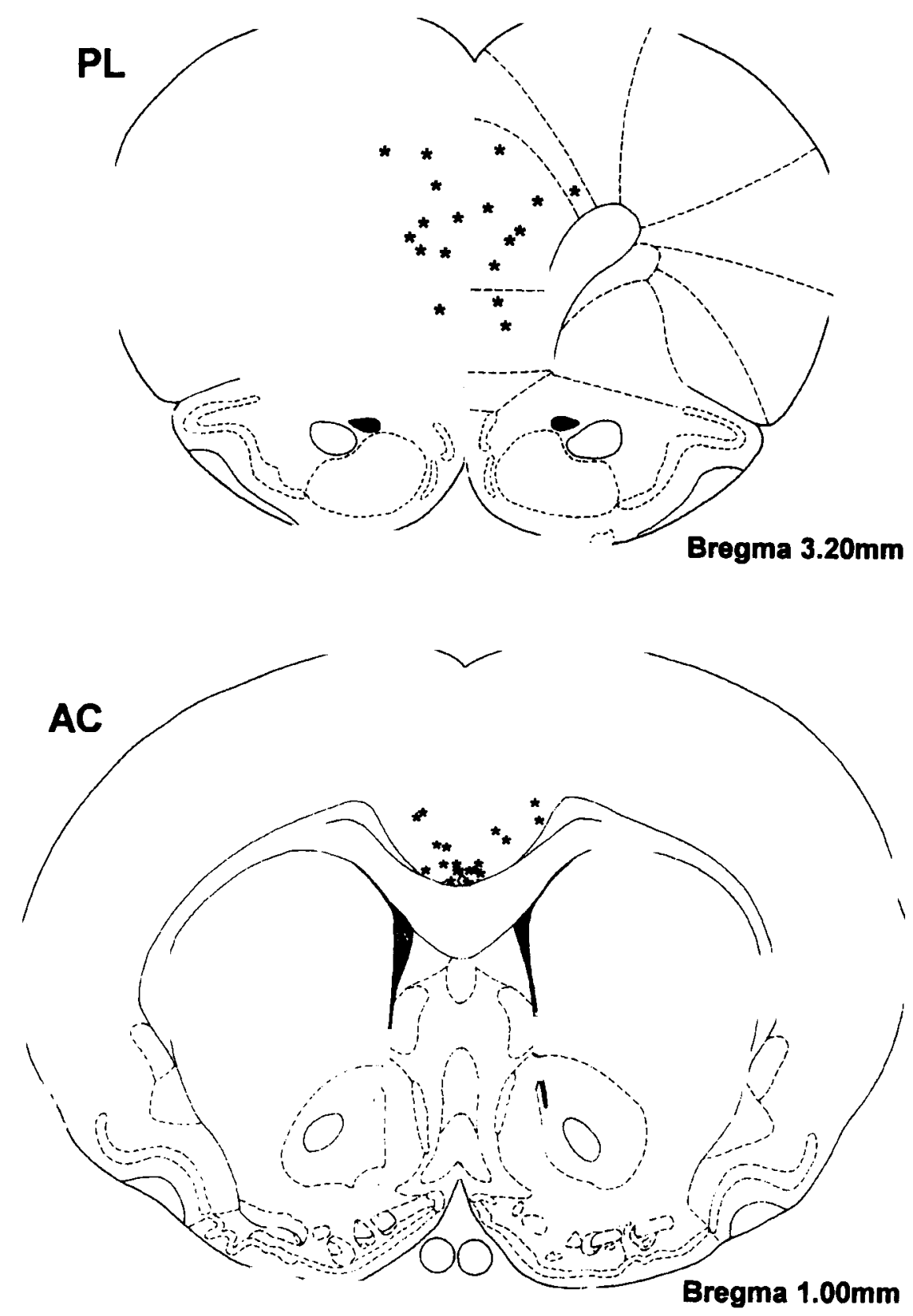

Figure 1. Schematics representing the areas of the medial prefrontal cortex with the heaviest concentrations of projected prelimbic $(P L)$ and post-genu anterior cingulate $(A C)$ injector tip placements. Top: The heaviest concentration of $P L$ injector tip placements was centered approximately $3.20 \mathrm{~mm}$ anterior to bregma. Bottom: The heaviest concentration of post-genu AC injector tip placements was centered approximately $1.00 \mathrm{~mm}$ anterior to bregma.

$p<.05]$ and several significant interaction effects, including infusion $\times$ group $[F(3,27)=6.119, p<.05]$, area $\times$ infusion $[F(3,27)=5.594, p<.05]$, area $X$ infusion $\times$ stimulus length $[F(3,27)=4.253, p<.05]$, and area $X$ infusion $\times$ group $[F(3,27)=3.052, p<.05]$. Further analyses revealed that these interactions were mainly due to impairments in the LDR group.

\section{Choice Accuracy Analysis in the LDB Group}

Analysis of the LDB data alone, using drug, infusion area, stimulus length, and stimulus length order as withinsubjects factors and overall percent accuracy as the dependent variable, revealed a significant stimulus length effect $[F(1,5)=73.382, p<.05]$ but no other significant effects or interactions, suggesting that the mPFC is not 
100

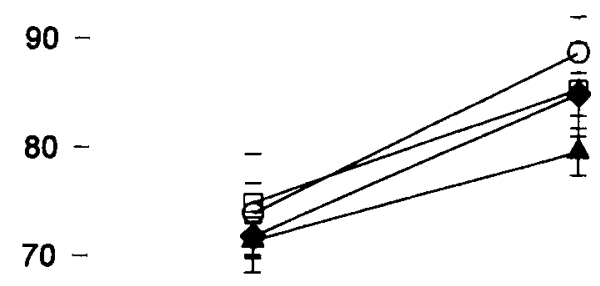

$60-$

$50-$

AC

40

200 msec

$500 \mathrm{msec}$
100

$90-$

$80-$

$70-$

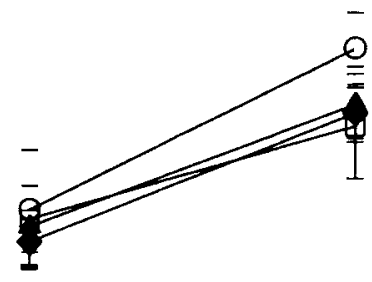

$60-$

$50-$

PL

40

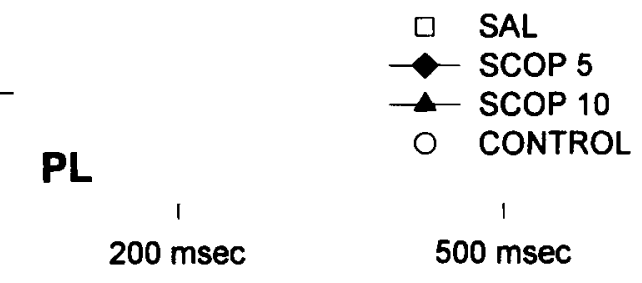

Stimulus Length

Stimulus Length

Figure 2. Infusions into the post-genu anterior cingulate (AC) cortex (left) and the prelimbic (PL) cortex (right) fail to impair performance on the light discrimination-block task $(p>.05)$.

actively involved in tasks in which the discriminability of the upcoming stimulus is predictable (Figure 2).

\section{Choice Accuracy Analysis in the LDR Group}

Analysis of the LDR data alone, using drug, infusion area, and stimulus length as within-subjects factors and overall percent accuracy as the dependent variable, revealed significant stimulus length effects $[F(1,4)=40.290$, $p<.05]$, with accuracy at the $200-\mathrm{msec}$ stimulus length consistently worse than that at the 500 -msec length, overall drug effects $[F(3,12)=12.364, p<.05]$, and a significant drug $\times$ area interaction $[F(3,12)=7.488, p<.05]$. An analysis of the effects of infusions into the $A C$ revealed that disruption of cholinergic transmission significantly impaired performance [main effect of drug: $F(3,12)=$ $12.998, p<.05]$. Infusions of both the $5-\mu \mathrm{g}$ and the $10-$ $\mu \mathrm{g}$ doses of scopolamine impaired performance $(p<.05)$, relative to saline infusions and to control days on the short stimulus length (200-msec) trials. On long stimulus length (500-msec) trials, only the $10-\mu \mathrm{g}$ dose of scopolamine impaired choice accuracy, relative to all the other conditions $(p<.05$; Figure 3$)$.

In the PL area, a repeated measures ANOVA, using drug and stimulus length as within-subjects factors and percent choice accuracy as the dependent measure, also revealed a significant overall drug effect $[F(3,12)=6.375$, $p<.05]$. Unlike the AC, the PL appeared to be sensitive to infusions in general. Saline infusions into the PL resulted in a decrement in choice accuracy at the long stimulus length $(p<.05)$. Infusions of the $10-\mu \mathrm{g}$ dose of scopolamine selectively impaired performance at the short stimulus length $(p<.05)$. Given the presence of saline infusion effects in the PL, the decrease in choice accuracy following infusions of the $10-\mu \mathrm{g}$ dose of scopolamine may be partially due to general effects of the infusion procedure. However, the decrement in performance at the short stimulus length following infusions of the $10-\mu \mathrm{g}$ dose of scopolamine was significantly greater than that seen following saline infusions $(p<.05$; Figure 3$)$.

\section{Omissions}

An analysis of omission rate data, using drug and infusion area as within-subjects factors and group as the between-subjects factor, revealed a significant overall group effect $[F(1,9)=16.325, p<.05]$, with omissions in all the drug conditions being higher in the LDR task than in the LDB task, and a general overall infusion effect $[F(3,27)=13.322, p<.05]$, with all infusions leading to higher omissions than did control days $(p<.05)$. Further analysis also revealed that omissions following infusions of the $10-\mu \mathrm{g}$ dose of scopolamine were significantly higher than omissions following the 5- $\mu \mathrm{g}$ dose of scopolamine $(p<.05)$. No other significant effects were found (see Table 1).

\section{Reaction Time}

An analysis of the reaction time data, using latency to respond following stimulus onset as the dependent variable, drug and infusion area as within-subjects factors, and group (LDB or LDR) as the between-subjects factor, revealed no significant main effects or interactions ( $p>$ .05 ). Thus, on trials in which a response occurred, the 
100

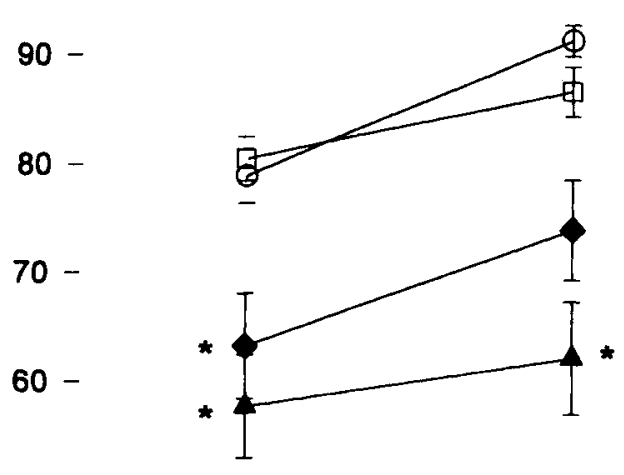

$50-$

AC

40

$$
\begin{gathered}
200 \mathrm{msec} \\
\text { Stimulus Length }
\end{gathered}
$$

100

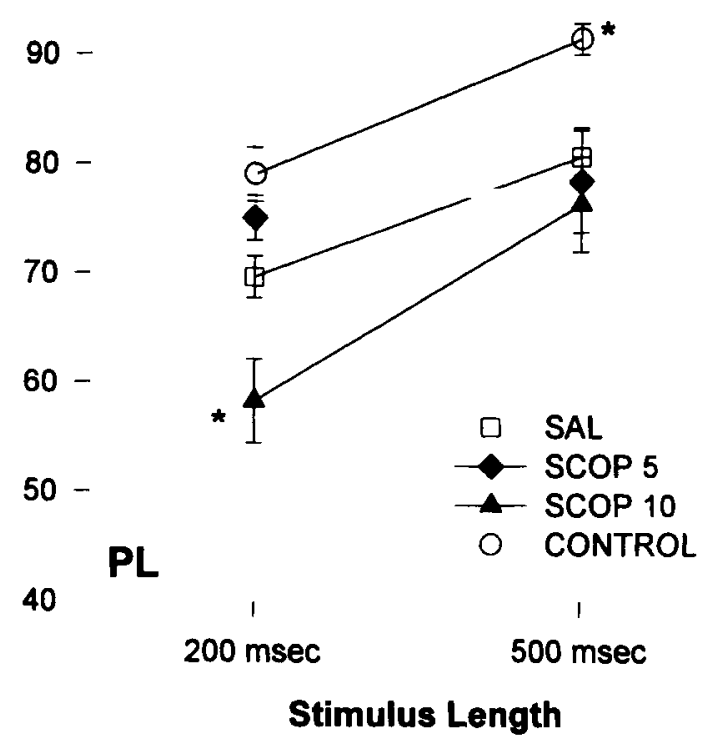

Figure 3. Infusions of scopolamine into the post-genu anterior cingulate (AC) cortex (left) and the prelimbic (PL) cortex (right) impair performance, relative to saline infusions, on the light discrimination-random task $(p<.05)$. ${ }^{D}$ ifferent from saline.

LDB and LDR groups, despite differences in choice accuracy, show similar reaction times across all drug conditions.

\section{DISCUSSION}

The present results suggest that the mPFC is not involved in all types of attentional processing. Rather, mPFC involvement is dependent on the predictability of the upcoming stimulus. The term predictability is often used in the attentional literature. For instance, in vigilance paradigms, the term often refers to the presentation of an oddball or a rare event. The term can also be used to describe the subject's ability to predict the location of an upcoming stimulus. In the present study, the term predictability is used in reference to the animal's ability to predict the stimulus length of an upcoming trial and, thus, how difficult the stimulus will be to detect.

The $\mathrm{mPFC}$ is not critical for maintaining optimal signal detection in a task (such as the LDB task) in which a rat can predict the difficulty level of the upcoming stimulus. In contrast, disruption of $\mathrm{mPFC}$ functioning significantly interferes with the ability to detect a light stimulus when the difficulty level of the upcoming stimulus is unpredictable (as in the LDR task). Infusions of scopolamine into the post-genu $\mathrm{AC}$ and the PL regions of the mPFC significantly impaired performance on the LDR, but not on the LDB task. This difference may be related to the dissociations seen in $\mathrm{mPFC}$ involvement in controlled versus automatic attentional processing.

In human subjects, the mPFC appears to be selectively engaged when task difficulty requires a more controlled or focused type of attentional processing. For instance, the mPFC becomes engaged when distracting stimuli are introduced or during learning of a new task. In the LDB task, the rats could predict the difficulty level of the upcoming stimulus. The repeated presentation of stimuli of the same stimulus length may have shifted the attentional demands from a more controlled type of attention to a more automatic type of attentional processing. The lower number of omissions in the LDB task (relative to omission rates in the LDR task) lends support to the hypothesis that the attentional demands of the LDB are less taxing than those required by the LDR task. The unpredictability of the LDR task may have required the rat to expend more attentional resources on accurate performance of the task.

However, the notion that the LDR task is more cognitively taxing than the LDB task appears to be contradicted by the overall level of choice accuracy. If the LDR task is indeed more cognitively taxing, one might expect LDR scores to be significantly lower in choice accuracy than those in the LDB task. This is not the case. There is no difference in choice accuracy under control or saline conditions between the two tasks. It is hypothesized that the rats adopt a strategy of maintaining an optimal level of performance. If a task is not cognitively challenging, the mPFC will not be activated, and performance will not be affected by prefrontal activity. In the less taxing task, involvement of the mPFC is not necessary to maintain an optimal level of performance. In contrast, if a task is more cognitively challenging (such as the LDR task), in order to maintain this same level of optimal performance, an intact $\mathrm{mPFC}$ is critical. The mPFC may allow an individual to maintain optimal performance during more challenging 
Table 1

Omission Rates and SEMs for the Light Discrimination-Random (LDR) and the Light-Discrimination-Block (LDB) Groups Following Infusions into the Anterior Cingulate and Prelimbic Regions

$\begin{array}{ccc}\text { Infusion } & \text { LDR } & \text { LDB } \\ \text { Control } & 26.00 \pm 3.5 & 11.70 \pm 4.1 \\ \text { line } & 35.85 \pm 5.4 & 21.65 \pm 4.4 \\ \text { copolamine, } 5 \mu \mathrm{g} & 32.65 \pm 4.0 & 23.50 \pm 3.5 \\ \text { copolamine, } 10 \mu \mathrm{g} & 41.10 \pm 2.8 & 26.45 \pm 3.3\end{array}$

Note-Omission rates are defined as the percent of total trials in which the rat failed to make a response. As there were no area effects, the anterior cingulate and prelimbic data were collapsed. Omission rates were higher in the LDR than in the LDB group and were higher following all infusions, as compared with control (no-infusion) sessions.

tasks by utilizing several mechanisms, including reducing the detrimental effects of distracting stimuli (Chao \& Knight, 1995; Sarter et al., 1996) or decreasing perseverative or premature responding (Muir et al., 1996).

Cholinergic input to the mPFC appears to play a vital role in maintaining optimal performance during controlled, but not during automatic attentional processing. In the LDB rats, infusions of the muscarinic antagonist scopolamine into the $\mathrm{AC}$ and $\mathrm{PL}$ regions did not significantly impair choice accuracy, relative to saline infusion or control days. In contrast, scopolamine infusions into the $\mathrm{AC}$ and the PL significantly decreased choice accuracy on the LDR task. In the LDR task, infusions of both the 5- $\mu \mathrm{g}$ and $10-\mu \mathrm{g}$ doses of scopolamine into the $\mathrm{AC}$ significantly decreased choice accuracy, relative to both saline infusion and control days. Despite the differences in omissions between the two tasks (omissions in the LDR task are significantly higher in all drug conditions, relative to the corresponding LDB conditions), it is unlikely that this dissociation in choice accuracy between the two tasks is due solely to motivational factors. On trials in which a response occurred, both LDR and LDB rats show similar latencies to respond to the light stimulus. Therefore, for the trials that are used to calculate choice accuracy, there is no significant motivation difference between the LDR and the LDB groups.

Similarly, the decrements in choice accuracy in the LDR task following scopolamine infusions into the $\mathrm{AC}$ are not simply due to a decreased motivation to perform. Reaction times were similar across all the drug conditions, and, although there was a general overall increase in omissions following all types of infusions (including saline infusions), omissions following scopolamine infusions were not significantly higher than those following saline infusions. Scopolamine infusions into the $\mathrm{AC}$ significantly impaired performance, whereas saline infusions did not, despite similar omission rates. Overall, the results suggest that cholinergic transmission within the $\mathrm{AC}$ is critical in detecting a light stimulus on tasks in which the difficulty level of an upcoming stimulus is unpredictable. Although the stimulus lengths used in the present study (200 and $500 \mathrm{msec}$ ) are longer than those used in other studies (e.g., Miner et al., 1997), the results suggest that the task parameters were sufficient to assess the rats' ability to detect the light stimulus. Choice accuracy was stimulus length dependent, with performance at the longer stimulus length being significantly better than performance at the short stimulus length.

In contrast to the results of infusions into the $\mathrm{AC}$, interpretation of the PL's role in attentional processing on the LDR task is less clear. Infusions of saline alone into the PL significantly impaired performance on the LDR task, relative to no-infusion sessions, indicating that the PL is very sensitive to the general infusion procedure. However, performance following infusions of scopolamine was significantly worse than that following saline infusions into the PL. Infusions of the $10-\mu \mathrm{g}$ dose of scopolamine significantly decreased choice accuracy at the short stimulus length, relative to saline infusions. These results suggest that muscarinic integrity within the PL is an important factor in mediating performance of the LDR task.

Although the decrement in choice accuracy following infusions into the PL may be the result of attentional deficits, the impairment could also be due to general behavioral disruption owing to motivational factors. The omission rate data suggests that motivational elements may indeed play a role. Infusions of saline and the $10-\mu \mathrm{g}$ dose of scopolamine into the PL, which both decreased choice accuracy, also both resulted in an increase in omission rates, as compared with control days. However, it should be noted that deficits in motivation were not invariably correlated with decrements in choice accuracy. In the LDB task, infusions into both the PL and the AC significantly increased omission rates without decreasing choice accuracy.

Although deficits in attention and/or motivation may account for the decrease in choice accuracy following infusions into the PL, there are other possible interpretations. For instance, it is possible that PL manipulations do not affect earlier, more attentional components of the LDR task, such as signal detection, but rather, later aspects of this task, such as decision-making processes. Ibotenic acid lesions of the MPFC (which included PL/IL cortices) did not result in disruption of sustained attentional processes (Miner et al., 1997). The excitotoxic lesions resulted in random leverpressing behaviors that were unaffected by traditional attentional manipulations, such as the presentation of distracting stimuli. The authors proposed that the mPFC lesions produced impairments in more "executive" processes, such as the ability to utilize proper task rules at the time a decision is required. Studies utilizing quinolinic acid lesions of the $\mathrm{MPFC}$ also support this conclusion. Although quinolinic acid lesions of the $\mathrm{mPFC}$ resulted in decrements in choice accuracy on a five-choice serial reaction time task, these excitotoxic lesions also appeared to result in increased perseverative responses and increased latencies to respond to a visual stimulus presented in one of five locations (Muir et al., 1996). Thus, the decrements in choice accuracy following lesions to the MPFC may be the result of impairments 
at the response or decisional stage of attentional tasks. Overall, although it is clear that the PL is involved in some aspect of attentional processing, the exact nature of the PL's involvement cannot be determined in the present study. Follow-up studies will be necessary to discern the specific contribution of the PL in attentional processing.

\section{REFERENCES}

Bussey, T. J., Muir, J. L., EveritT, B. J., \& Robbins, T. W. (1997) Triple dissociation of anterior cingulate, posterior cingulate, and medial frontal cortices on visual discrimination tasks using a touch screen testing procedure for the rat. Behavioral Neuroscience, 111, 920- 936.

ChaO, L. L., \& KNIGHT, R. T. (1995). Human prefrontal lesions increase destructibility to irrelevant sensory inputs. NeuroReport, 6, 1605-1610.

Corbetta, M., Miezin, F. M., Dobmeyer, S., Shulman, G. L., \& PeTERSEN, S. E. (1991). Selective and divided attention during visual discriminations of shape, color, and speed: Functional anatomy by positron emission tomography. Journal of Neuroscience, 11, 2383-2402.

Delatour, B., \& Gisquet-Verrier, P. (1996). Prelimbic cortex specific lesions disrupt delayed-variable response tasks in the rat. Behavioral Neuroscience, 6, 1282-1298.

Gevins, A., Smith, M. E., McEvoy, L., \& Yu, D. (1997). Highresolution EEG mapping of cortical activation related to working memory: Effects of task difficulty, type of processing, and practice. Cerebral Cortex, 7, 374-385.

Granon, S., Poucet, B., Thinus-Blanc, C., Changeux, J. P., \& VIDAL, C. (1995). Nicotinic and muscarinic receptors in the rat prefrontal cortex: Differential roles in working memory, response selection and effortful processing. Psychopharmacology, 119, 139-144.

Herremans, A. H. J., Hijzen, T. H., \& Olivier, B. (1997). Effects of cholinergic drug infusions into the dorsal part of the medial prefrontal cortex on delayed conditional discrimination performance in the rat. Behavioural Brain Research, 84, 291-299.

Herremans, A. H. J., Hijzen, T. H., Welborn, P. F. E., Olivier, B., \& SLANGEN, J. L. (1996). Effects of infusion of cholinergic drugs into the prefrontal cortex area on delayed matching to position performance in the rat. Brain Research, 711, 102-111.

Jenkins, I. H., Brooks, D. J., Nixon, P. D., Frackowiak, R. S., \& PassINGHAM, R. E. (1994). Motor sequence learning: A study with positron emission tomography. Journal of Neuroscience, 14, 3775-3790.

Jueptner, M., Stephan, K. M., Frith, C. D., Brooks, D. J., Frackowiak, R. S., \& Passingham, R. E. (1997). Anatomy of motor learning: I. Frontal cortex and attention to action. Journal of Neurophysiology, 77, 1313-1324
LECAS, J. C. (1995). Prefrontal neurones sensitive to increased visual attention in the monkey. NeuroReport, 7, 305-309.

MCGaughy, J., Kaiser, T., \& SARTER, M. (1996). Behavioral vigilance following infusions of 192 IgG-saporin into the basal forebrain: Selectivity of the behavioral impairment and relation to cortical AChEpositive fiber density. Behavioral Neuroscience, 110, 1-19.

Mesulam, M.-M., Mufson, E. J., Levey, A. I., \& Wainer, B. H. (1983). Cholinergic innervation of cortex by the basal forebrain: Cytochemistry and cortical connections of the septal area, diagonal band nuclei, nucleus basalis (substantia innominata), and hypothalamus in the rhesus monkey. Journal of Comparative Neurology, 214, 170-197.

Miner, L. H., Ostrander, M., \& Sarter, M. (1997). Effects of ibotenic acid-induced loss of neurons in the medial prefrontal cortex of rats on behavioral vigilance: Evidence for executive dysfunction. Journal of Psychopharmacology, 11, 169-178.

Muir, J. L., EveritT, B. J., \& RobBins, T. W. (1994). AMPA-induced excitotoxic lesions of the basal forebrain: A significant role for the cortical cholinergic system in attentional functioning. Journal of Neuroscience, 14, 2313-2326.

Muir, J. L., EveritT, B. J., \& Robrins, T. W. (1996). The cerebral cortex of the rat and visual attentional function: Dissociable effects of mediofrontal, cingulate, anterior dorsolateral, and parietal cortex lesions on a five-choice serial reaction time task. Cerebral Cortex, 6 , 470-481.

Pardo, J. V., Pardo, P. J., Janer, K. W., \& Raichle, M. E. (1990). The anterior cingulate cortex mediates processing selection in the Stroop attentional conflict paradigm. Proceedings of the National Academy of Sciences, 87, 256-259.

Passingham, R. E. (1996). Attention to action. Philosophical Transactions of the Royal Society of London: Series B, 351, 1473-1481.

Petersen, S. E., Van Mier, H., Fiez, J. A., \& Raichle, M. E. (1998). The effects of practice on the functional anatomy of task performance. Proceedings of the National Academy of Sciences, 95, 853 860.

Ragozzino, M. E., Adams, S., \& Kesner, R. P. (1998). Differential involvement of the dorsal anterior cingulate and prelimbic-infralimbic areas of the rodent prefrontal cortex in spatial working memory. Behavioral Neuroscience, 112, 293-303.

Sarter, M., Bruno, J. P., Givens, B., Moore, H., McGaughy, J., \& MCMAHON, K. (1996). Neuronal mechanisms mediating drug-induced cognition enhancement: Cognitive activity as a necessary intervening variable. Cognitive Brain Research, 3, 329-343.

(Manuscript received October 15, 1998; revision accepted for publication August 3, 1999.) 